\title{
Combined Use of the Illizarov Method, Concentrated Bone Marrow Aspirate
}

\author{
Edgardo R Rodriguez Collazo* \\ Chicago Foot and Ankle Deformity Correction Center, USA
}

Submission: October 14, 2015; Published: October 28, 2015

*Corresponding author: Edgardo R. Rodriguez, Chicago Foot and Ankle Deformity Correction Center; Chicago, Illinois, Department of Surgery, Saint Joseph Hospital Resurrection Health Care; Saint Anthony Hospital of Chicago, USA, Tel: 312-335-3939; Fax: 312-284-4892; Email: egodpm@gmail.com

\section{Abstract}

Background: Non-union fractures, particularly in patients experiencing soft-tissue loss, comorbidities, and chronic infection, can be difficult to treat. These fractures are typically accompanied by high rates of post-surgical non-union and recurrence of infection. The Ilizarov method has addressed conditions such as infection, deformity, soft-tissue loss, and joint contracture. However, techniques to optimize the osteogeneic potential of inured bone are relatively new. Autologous concentrated bone marrow aspirate (cBMA) and Platelet Rich Plasma (PRP) are therapies that increase osteogeneic potential of osteoconductive demineralized bone matrix (DBM).

Purpose: To evaluate the efficacy of the Ilizarov method combined with a cocktail of demineralized bone matrix (DBM), concentrated cBMA and PRP in promoting healing of bone.

Study Design: A retrospective case series.

Patient Sample: Thirteen patients (N=13, 10 men, 3 women), mean age 52.9 years with comorbidities (diabetes, obesity, smoking, renal disease) and closed bimalleolar ( $\mathrm{N}=10)$, pilon $(\mathrm{N}=2)$, or distal tibia $(\mathrm{N}=1)$ fractures.

Methods: Ilizarov method and a combination of DBM, cBMA (30 ml) and PRP (30 ml).

Outcome Measures: Time on fixator, incidence of infection, and radiographs to assess fracture repair.

Results: Post-Operative radiographs for 11 of 13 patients showed complete fracture repair when the external fixator was removed $(\sim$ four months post-surgery). One of the two patients who failed to heal with the first treatment received an additional cBMA treatment and healed. The second case was not painful, and eventually healed without residual deformity. No infections or amputations occurred in this case series.

Conclusion: Use of DBM, cBMA and PRP in combination with the Ilizarov method results in an $85 \%$ success rate for fracture healing within approximately four months. We conclude that the Ilizarov method combined with DBM, cBMA and PRP is a safe, reliable, and effective method with good clinical outcomes for the treatment of non-union fractures in patients with significant co-morbidities. The addition of PRP and cBMA decreased treatment time, reduced complication rate, and incidence of infection.

Keywords: Co-morbidities; Osteoblasts; Bone grafts

Abbreviations: cBMA: Concentrated Bone Marrow Aspirate; PRP: Platelet Rich Plasma; DBM: Demineralized Bone Matrix; ICBG: Iliac Bone Graft; AOPER: AO Pronation External Rotation; ACD-A: Anticoagulant Citrate Dextrose Solution

\section{Introduction}

Serious orthopedic injuries such as non-unions and bimalleolar and pilon fractures, can be treated successfully with the Ilizarov method [1]. However, complications to include contractures, pin track infections, loss of range of motion, and articular damage can occur. Patients who are experiencing softtissue loss, comorbidities, and chronic infection, increase the complexity of these cases. Patients who are smokers of advanced age with a high rate of systemic illness are the most difficult to treat because these factors negatively impact the rate of healing and incidence of infection $[2,3]$.
Therefore, the overarching challenge that surgeons face with these patients is to achieve a stable, well-aligned, mobile and pain-free joint while minimizing the risk of infection and post-traumatic osteoarthritis. The classic Illizarov technique has several advantages when treating patients with various comorbidities [4]. With this technique, the reduction and fixation of the fracture is performed with minimal soft-tissue exposure and blood loss and the fixation is stable enough to allow weight bearing soon after surgery. The external fixator affords the surgeon the opportunity to adjust the alignment, compression, and distraction during surgery and while the patient heals. 


\section{Orthopedics and Rheumatology Open Access Journal}

Further, screws and plates are not left behind once the fracture is healed. Collectively, these factors improve the success rate of the Ilizarov method in fracture repair, and have a positive effect on patient quality of life [5].

In the treatment of complicated fractures, surgeons may also use an autologous iliac bone graft (ICBG) to augment healing potential. Specifically, autologous bone grafts possess the three key properties required for bone growth: osteogenic, osteoinductive and osteoconductive. Unfortunately, in patients with multiple co-morbidities, autologous bone grafting may introduce new complications, particularly at the donor site. These include bleeding, infection, and chronic pain. Moreover, limited bone graft material is available which may not be sufficient for treatment [6]. Since the success of a bone graft is determined by the ability for the grafted tissue to recruit progenitor cells to the injured area to form osteoblasts for healing purposes, alternative methods are needed in compromised patients.

Safe and effective alternative graft materials have become increasingly popular for use in patients with significant comorbidities. Demineralized bone matrix (DBM) is donor tissue that has been processed to remove inorganic mineral content, resulting in an organic collagen matrix [7]. As a result of this processing, DBM only retains osteoinductive properties, highlighting the need for an additional material that can provide osteoprogenitor cells to improve the osteogeneic environment. One strategy is to aspirate the patient's own bone marrow, remove red blood cells and concentrate osteoprogenitor cells in each volume of blood harvested. The benefit of this is twofold: red blood cells can interfere with bone formation and healing [5] and concentrated osteoprogenitor cells increase the osteogeneic environment (Figures 1 \& 2).

Consequently, the use of concentrated Bone Marrow Aspirate (cBMA) combined with an osteoinductive bone graft substitute (DBM) insures that two of the main properties for bone growth and healing are present with minimal risk and morbidity to the patient. Additionally, the use of Platelet Rich Plasma (PRP), a concentrated portion of a patient's own blood, can augment the healing process since it introduces various growth factors and cytokines that can reduce infection and promote regenerative processes [8].

This case series retrospectively analyzes the safety and efficacy of combining DBM, cBMA, PRP, and the Ilizarov technique to promote fracture healing in a population of patients with complicated fractures and significant comorbidities.

\section{Materials and Methods \\ Patient Demographics}

Thirteen patients $(\mathrm{N}=13)(\mathrm{N}=10$ men and 3 women), mean age 52.9 years) with various comorbidities (diabetes, obesity, smoking, renal disease). All patients had closed bimalleolar $(\mathrm{N}=10)$, pilon $(\mathrm{N}=2)$, or distal tibia $(\mathrm{N}=1)$ fractures with a poor soft tissue envelope. Of the bimalleolar fractures, seven were AO Supination External Rotation (AOSER), and three were AO Pronation External Rotation (AOPER). The pilon Fractures were AO classification Type 1 Ruedi and Allgower, respectively. The
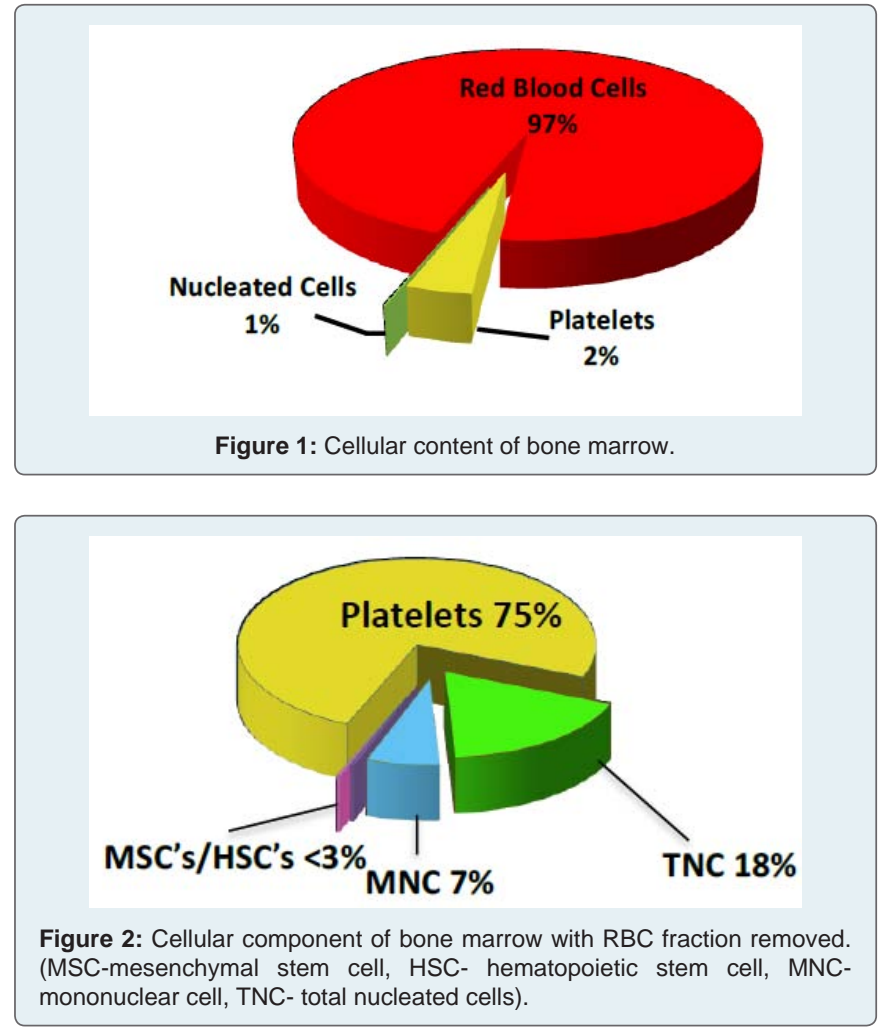

distal tibia fracture was a Type 2 fracture. Patients included in this case series received implantation of cBMA, PRP, and DBM mixture along with Ilizarov fixation between January 2012 and December 2012. Patients were identified by a retrospective review of physician office records.

As illustrated in Table 1, all patients had multiple comorbidities including obesity $(\mathrm{N}=13)$; diabetes $(\mathrm{N}=12)$; smoking $(\mathrm{N}=8)$; osteoporosis $(\mathrm{N}=3)$, renal disease $(\mathrm{N}=3)$ and abnormal bone metabolic panels (Vitamin D and Calcium deficiency, $\mathrm{N}=10$ ).

\section{Bone Marrow Aspiration and Concentration}

In all 13 procedures, $26 \mathrm{ml}$ of bone marrow was harvested from the medial aspect of the proximal tibia (Figure 3A). First, the $30 \mathrm{ml}$ syringe was primed with $4 \mathrm{ml}$ of anticoagulant citrate dextrose solution (ACD-A) (Arteriocyte Medical Systems, Hopkinton, MA). A mallet was then used to advance the 15 gauge Jamshidi needle (Arteriocyte Medical Systems, Hopkinton, MA) until a decrease in resistance was met. The decreased resistance was used to indicate that the needle had entered the marrow cavity. Bone marrow was then drawn back gently (to prevent lysing of the cells) into the $30 \mathrm{ml}$ syringe that was primed with $4 \mathrm{ml}$ of ACD-A. During the marrow draw, if significant resistance was met, the needle was repositioned until the marrow flowed easily. This prevented clotting and unnecessary physical disruption of the cBMA, which can lyse cells and reduce growth factor content of the platelets. The needle was repositioned until a total volume of $30 \mathrm{ml}$, including the ACD-A, was harvested.

The harvested marrow was then filtered and processed in the Arteriocyte Magellan system (Arteriocyte Medical Systems, Inc., Hopkinton, MA) (Figure 3B). The Magellan is an automatic, 


\section{Orthopedics and Rheumatology Open Access Journal}

Table 1: Patient Demographics.

\begin{tabular}{|c|c|c|c|c|}
\hline Patient & Fracture & Gender & Age & Comorbidity \\
\hline & Type & & & \\
\hline \multirow[t]{2}{*}{1} & Bimalleolar & M & 52 & Smoking, \\
\hline & & & & Diabetes, Obesity \\
\hline \multirow[t]{2}{*}{2} & Bimalleolar & M & 47 & Smoking, \\
\hline & & & & Diabetes, Obesity \\
\hline 3 & Bimalleolar & M & 63 & Diabetes, Obesity \\
\hline \multirow[t]{2}{*}{4} & Bimalleolar & M & 55 & Smoking, \\
\hline & & & & Diabetes, Obesity \\
\hline \multirow[t]{4}{*}{5} & Bimalleolar & M & 68 & Smoking, \\
\hline & & & & Diabetes, \\
\hline & & & & Obesity, \\
\hline & & & & Renal Disease \\
\hline 6 & Bimalleolar & M & 49 & Diabetes, Obesity \\
\hline \multirow[t]{4}{*}{7} & Bimalleolar & $\mathrm{W}$ & 62 & Smoking, \\
\hline & & & & Diabetes, \\
\hline & & & & Obesity, \\
\hline & & & & Osteoporosis \\
\hline \multirow[t]{3}{*}{8} & Bimalleolar & M & 56 & Diabetes, \\
\hline & & & & Obesity, \\
\hline & & & & Renal Disease \\
\hline \multirow[t]{3}{*}{9} & Bimalleolar & M & 39 & Smoking, \\
\hline & & & & Diabetes, \\
\hline & & & & Obesity \\
\hline \multirow[t]{2}{*}{10} & Bimalleolar & M & 51 & Diabetes, \\
\hline & & & & Obesity \\
\hline \multirow[t]{4}{*}{11} & Pilon & M & 65 & Smoking, \\
\hline & & & & Diabetes, \\
\hline & & & & Obesity, \\
\hline & & & & Renal Disease \\
\hline \multirow[t]{4}{*}{12} & Pilon & $\mathrm{W}$ & 54 & Smoking, \\
\hline & & & & Diabetes, \\
\hline & & & & Obesity, \\
\hline & & & & Osteoporosis \\
\hline \multirow[t]{3}{*}{13} & Distal Tibia & W & 27 & Pre-Diabetes, \\
\hline & & & & Obesity, \\
\hline & & & & Osteoporosis \\
\hline
\end{tabular}

dual spin, closed system allowing for safe and rapid bedside concentration of whole blood and bone marrow aspirate. For each case, the Magellan was customized to obtain a final volume of cBMA of approximately $3 \mathrm{ml}$ from $30 \mathrm{ml}$ of bone marrow aspirate.

\section{Blood Draw for Concentration to Platelet Rich Plasma}

For each patient, $30 \mathrm{ml}$ of blood was drawn from an antecubital vein. First, the $30 \mathrm{ml}$ syringe was primed with $4 \mathrm{ml}$ of ACD-A and then $26 \mathrm{ml}$ of blood was obtained from each patient using standard phlebotomy procedures. Once the final volume of $30 \mathrm{ml}$ was obtained, the syringe was loaded into the Magellan System. The Magellan was programmed to produce $3 \mathrm{ml}$ of Platelet Rich Plasma (PRP) from the $30 \mathrm{ml}$ volume (Figure 3B).

\section{Demineralized Bone Matrix (DBM)}

Integra Accell EVO, which is a DBM and poloxamer Reverse
Phase Medium produced by Integra (Irvine, CA) was used in all patients. This form of DBM provides a high surface area, which facilitates binding access for natural bone proteins, providing a scaffold and signal for new bone formation. The Accell EVO DBM is moldable putty at room temperature, which becomes more viscous at body temperature. Prior to use, the Accell EVO DBM was mixed with the cBMA to achieve desired consistency (Figure 3C \& 3D). Five cc's of DBM were injected into areas of non-union in patients with bimalleolar and distal tibia fractures. Ten cc's of the Accell Evo DBM were used for pilon fractures (Figure 3E).

\section{Ilizarov Technique}

The circular fixator consisted of a pre-constructed frame that consisted of four rings (Figure 4). A proximal reference wire was fixed and tensioned to the most proximal ring. A distal reference wire was fixed directly proximal to the ankle. After the bone ends were fixed with opposing olive wires, radiology was used to ascertain alignment of the bone. In cases where the non-union was close to the ankle, the foot was incorporated into the frame to enhance stability and prevent equinus or varus contracture. When the bones were properly aligned, the cBMA, PRP, and Accell EVO DBM mixture was injected percutaneously using the 15-gauge bone marrow aspiration needle (Figure 3E). Any remaining cBMA and PRP was injected directly into the joint space. Viability of the foot was assessed via palpation of the

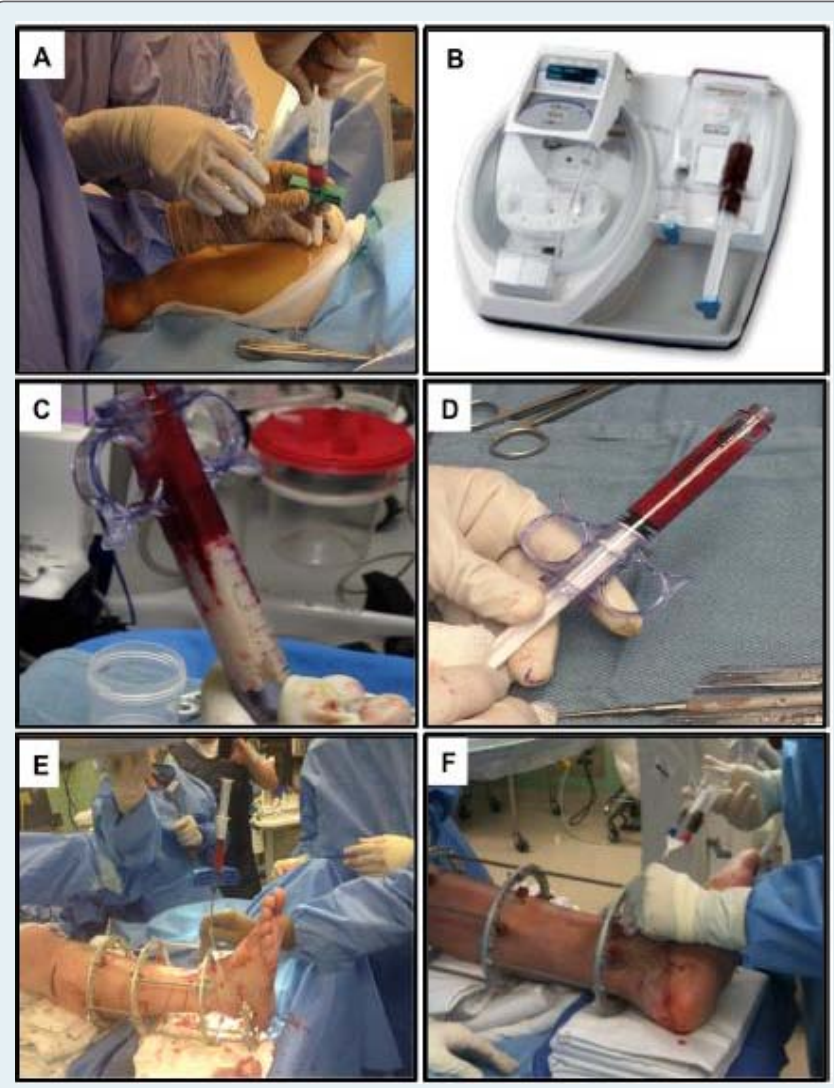

Figure 3: A. Bone marrow aspiration method. B. Magellan Platelet Separator. C. Syringe containing initial mixture of cBMA and DBM. D. Final implantable mixture of $C B M A$ and DBM . E. Application of $c B M A$ and graft material to fracture site. F. Application of PRP using a spray tip cannula. 


\section{Orthopedics and Rheumatology Open Access Journal}

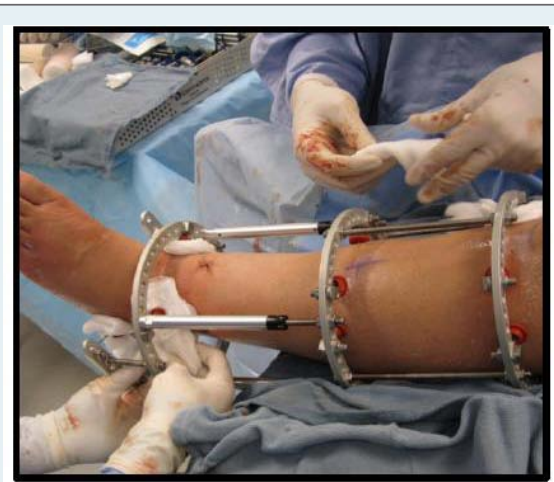

Figure 4: llizarov method of fixation.

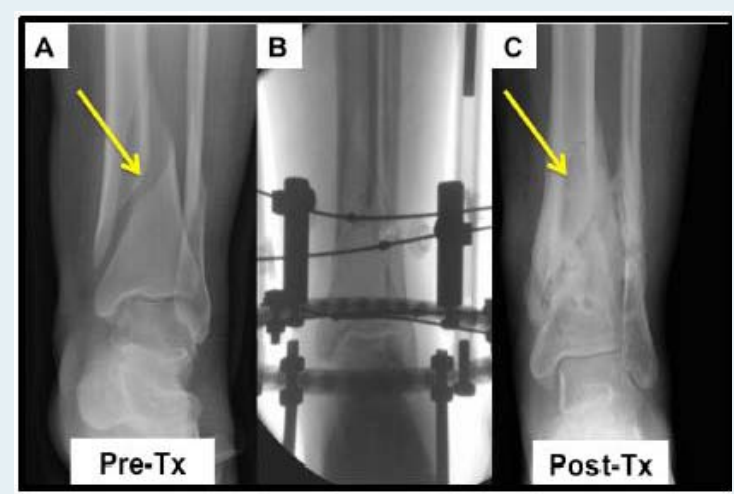

Figure 5: A. Pre-treatment radiograph of a female patient with a distal tibial fracture (yellow arrow), B. Fluoroscopy image of olive wire stabilization of the fracture. C. Post-treatment radiographs of same female patient at 16 weeks post-procedure. Fracture is healed (yellow arrow).

dorsalis pedis and posterior tibial pulses, and measurement of oxygen saturation of the hallux. If circulation appeared to be disrupted, the length was adjusted as necessary to return circulation to normal. PRP combined with calcium chloride and thrombin solution, was applied using a spray-tip cannula at all wound sites to expedite healing (Figure 3F).

\section{Results}

Mean fixator time was 16 +/- 2 weeks. Post-operative radiographs for eleven of thirteen patients showed complete bone healing at the time that the external fixator was removed (approximately 4 months) (Figure 5). No infections were reported and no limbs were amputated in this series.

Patient 4 and Patient 7 experienced delayed closure. Patient 4 required a revision due to consistent pain. Four weeks postrevision, pain had subsided. Patient 7 had consistent pain postprocedure. A revision was performed with a percutaneous injection of cBMA. This patient was pain free within four weeks post-revision. Following revision, both patients healed within approximately four months without residual deformity or morbidity.
Three patients experienced mild adverse reactions to include angulation of the fracture site $(\mathrm{N}=1)$ and superficial irritation due to wires $(\mathrm{N}=2)$, which did not require antibiotics. Both pilon fractures had transient traction neuropathy of the superficial peroneal nerve.

\section{Summary}

The aim of the author's strategy in combining the Ilizarov technique with DBM, cBMA, and PRP was to decrease the period of external fixation, expedite fracture healing, and diminish the rate of complications, specifically infections. In this case series, complications that were reported were minor and only one required additional surgery. Excellent results were obtained in 11 of 13 patients and good results were obtained in the other two patients following additional interventions.

In summary, use of DBM, saturated with cBMA and PRP, in combination with the Ilizarov technique results in an $85 \%$ healing rate in approximately four months. We conclude that the Ilizarov method combined with cBMA and PRP is a safe, reliable, and effective method with good clinical outcomes for the treatment of non-union fractures in patients with significant co morbidities. The addition of cBMA resulted in a shorter treatment time and reduced complication rate. The addition of PRP, particularly to wound sites, reduced incidence of infection and promoted cutaneous tissue healing.

\section{References}

1. Krappinger D, Irenberger A, Zegg M, Huber B (2013) Treatment of large posttraumatic tibial bone defects using the Ilizarov method: a subjective outcome assessment. Arch Orthop Trauma Surg 133(6): 789-795.

2. Blum AL, BongioVanni JC, Morgan SJ, Flierl MA, dos Reis FB (2010) Complications associated with distraction osteogenesis for infected nonunion of the femoral shaft in the presence of a bone defect: a retrospective series. J Bone Joint Surg Br 92(4): 565-570.

3. Sella EJ (2008) Prevention and management of complications of the Ilizarov treatment method. Foot Ankle Spec 1(2): 105-107.

4. Ramos T, Karlsson J, Eriksson BI, Nistor L (2013) Treatment of distal tibial fractures with the Ilizarov external fixator--a prospective observational study in 39 consecutive patients. BMC Musculoskelet Disord 14: 30 .

5. Brinker MR, O'Connor DP (2007) Outcomes of tibial nonunion in older adults following treatment using the Ilizarov method. J Orthop Trauma 21(9): 634-642.

6. Pieske O, Wittmann A, Zaspel J, Löffler T, Rubenbauer B, et al. (2009) Autologous bone graft versus demineralized bone matrix in internal fixation of ununited long bones. J Trauma Manag Outcomes 3: 11.

7. Gruskin E, Doll BA, Futrell FW, Schmitz JP, Hollinger JO (2012) Demineralized bone matrix in bone repair: history and use. Advanced drug delivery reviews 64(12): 1063-1077.

8. Guo Y, Qiu J, Zhang C (2008) Follow-up study on platelet-rich plasma in repairing chronic wound nonunion of lower limbs in 47 cases. Zhongguo Xiu Fu Chong Jian Wai Ke Za Zhi 22(11): 1301-1305. 